\title{
Temperature Dependence of Interfacial Tension of Demixed Polymer Blend Melts: Polystyrene/Poly(dimethylsiloxane)s
}

\author{
Takuhei NosE \\ Department of Polymer Chemistry, Tokyo Institute of Technology, \\ O-okayama, Meguro-ku, Tokyo 152, Japan
}

(Received July 10, 1996)

\begin{abstract}
Interfacial tension of polystyrene/poly(dimethylsiloxane) blends with different molecular weights have been measured as a function of temperature by sessile drop method. The results are described as a scaled relation of reduced interfacial tension $v$ s. reduced segregation strength, $\chi / \chi_{\mathrm{c}}^{-1}$, where $\chi$ is the segmental interaction parameter and $\chi_{\mathrm{c}}$ is $\chi$ at the critical temperature. The scaled relation is discussed on the basis of mean-field theory, and a semi-empirical expression has been presented for the scaled relation covering a wide temperature range.

KEY WORDS Interfacial Tension / Polymer Blends / Temperature Dependence / Polystyrene / Poly(dimethylsiloxane)/
\end{abstract}

It is theoretically predicted that there are three temperature regions for interfacial tension of polymer melts according to the segregation strength. ${ }^{1,2}$ In Region I of weak segregation near the critical point, the interfacial tension appears and increases as temperature goes away from the critical point. Region I is specified by $\chi \cong 2 / N$, where $\chi$ is the Flory-Huggins interaction parameter and $N$ is the degree of polymerization. In strong-segregation region, Region II, where $2 / N<\chi<1$, the interfacial tension is proportional to $T \chi^{1 / 2}$, while in Region III of very strong segregation, where $\chi>1$, it is proportional to $T \chi$, with $T$ being the absolute temperature. A region unique to polymeric systems is Region II, where the interfacial tension can increase with increasing temperature even if the polymer blend has the upper critical solution temperature (UCST).

In our previous studies, Region I and Region II have been investigated by measuring the temperature dependence of interfacial tension for polystyrene (PS)/ poly(dimethylsiloxane) (PDMS) blends. ${ }^{3,4}$ It has been demonstrated that the interfacial tension exhibits predicted temperature dependences unique to Region I and Region II, respectively. In this study, adding data of interfacial tension in the intermediate region, we will make a better understanding of the interfacial-tension behavior over the whole temperature region from Region I to Region II by scaled plots.

The author has measured coexistence curves of PS/ PDMS mixtures with different molecular weights to evaluate the interaction parameter $\chi$ as functions of temperature and molecular weight. ${ }^{5}$ The value of $\chi$ is indispensable and will be utilized in data analysis and discussion in the present study. The same series of blend systems are here used to study the intermediate region. Details of the scaling arguments based on the squaregradient theory of mean-field type for the interfacial tension will be made, and a semi-empirical expression will be presented.

\section{THEORETICAL CONSIDERATIONS AND A SCALED EXPRESSION}

Adopting the square-gradient theory for the free energy of a non-uniform binary system, the interfacial tension $\gamma$ is given by ${ }^{1,2,6}$

$$
\gamma=\int_{-\infty}^{\infty}\left[\Delta f(\phi)+\kappa(\partial \phi / \partial z)^{2}\right] \mathrm{d} z / v_{0}
$$

Here, $\Delta f$ is the excess local free energy per site as a function of concentration $\phi$ in volume fraction at the position $z$ with the $z$-axis being perpendicular to the interface, $\kappa$ is the coefficient of square-gradient term, and $v_{0}$ is the site volume. Starting with eq 1 , one has the following equations held at equilibrium.

$$
\begin{gathered}
\Delta f(\phi)=\kappa(\partial \phi / \partial z)^{2} \\
\gamma=\left(2 / v_{0}\right) \int_{-\infty}^{\infty} \Delta f(\phi) \mathrm{d} z \\
=\left(2 / v_{0}\right) \int_{\phi^{\prime}}^{\phi^{\prime \prime}}[\kappa \Delta f(\phi)]^{1 / 2} \mathrm{~d} \phi
\end{gathered}
$$

An interfacial thickness $L$ is defined as

$$
L=\Delta \phi /\left(\frac{\partial \phi}{\partial z}\right)_{\max }=[\kappa / \Delta f(\phi)]_{\max }^{1 / 2} \Delta \phi
$$

with $\Delta \phi$ being difference in concentration between coexisting phases, and the subscript, max, denotes the value at the maximum of concentration gradient at interface. Adopting an approximation $[\Delta f(\phi)]_{\max }=$ $-S_{\max }(\Delta \phi)^{2} / 8$ with

$$
S=\left(\partial^{2} \Delta f / \partial \phi^{2}\right)
$$

one can obtain the following approximate expressions for interfacial tension $\gamma$ and the interfacial thickness $L$ from eq 3 and $4 .^{1}$

$$
\begin{aligned}
\gamma & =[\Delta f(\phi)]_{\max } L / v_{0} \\
& =\kappa_{\max }\left(-S_{\max }\right)^{1 / 2}(\Delta \phi)^{2} /\left(2 \sqrt{2} v_{0}\right)
\end{aligned}
$$

and

$$
L=2 \sqrt{2} \kappa_{\max }^{1 / 2}\left(-S_{\max }\right)^{-1 / 2}
$$

For a binary polymer blend, $S$ and $\kappa$ are given in the Flory-Huggins type mean-field theory as ${ }^{7}$ 


$$
\begin{aligned}
\Delta f= & \Delta_{\mathrm{m}} f-\sum_{i=1}^{2} \phi_{i} \Delta \mu_{i}(e) \\
= & \frac{\phi_{1}}{N_{1}} \ln \left(\phi_{1} / \phi_{1}(e)\right)+\frac{\phi_{2}}{N_{2}} \ln \left(\phi_{2} / \phi_{2}(e)\right) \\
& -\frac{1}{N_{1}}\left(\phi_{1}-\phi_{1}(e)\right)-\frac{1}{N_{2}}\left(\phi_{2}-\phi_{2}(e)\right) \\
& +\chi\left(\phi_{1}-\phi_{1}(e)\right)\left(\phi_{2}-\phi_{2}(e)\right) \\
& \quad S=\frac{1}{N_{1} \phi_{1}}+\frac{1}{N_{2} \phi_{2}}-2 \chi \\
\kappa= & \kappa_{\mathrm{e}}+\kappa_{\mathrm{s}} \\
= & k T \chi \lambda^{2} / 2+k T\left(l_{1}^{2} / \phi_{1}+l_{2}^{2} / \phi_{2}\right) / 36
\end{aligned}
$$

Here, $\Delta_{\mathrm{m}} f$ is the free energy of mixing, $\mu(e)$ is the chemical potential, and $(e)$ denotes the value at equilibrium (in bulk phase). The subscripts 1 and 2 denote the constitutional polymers 1 and 2 , respectively, $N$ is the polymeric index, $\chi$ is the interaction parameter per site between polymer 1 and polymer $2, l$ is the Kuhnsegment length, and $\lambda$ represents the range of segmentsegment interactions. $\kappa_{\mathrm{e}}$ and $\kappa_{\mathrm{s}}$ are energy and entropy terms in $\kappa$, corresponding to the first and the second terms on the right hand side of eq 10 , respectively.

Hereafter for a while, let us suppose a symmetrical system with $N_{1}=N_{2}=N$ and $l_{1}=l_{2}=l$, for simplicity. The critical point is located at $\chi=2 / N=\chi_{\mathrm{cm}}$, and $-S_{\max }$ and $\kappa_{\max }$ are given from eq 9 and 10 by putting $\phi=1 / 2$ as

$$
\begin{gathered}
-S_{\max }=2 k T\left(\chi-\chi_{\mathrm{cm}}\right) \\
\kappa_{\max }=k T\left(\chi \lambda^{2} / 2+l^{2} / 9\right)
\end{gathered}
$$

In Region I (weak segregation; near critical, $\chi \sim \chi_{\mathrm{cm}}=$ $2 / N)$, one has $\kappa_{\max }=k T l^{2} / 9$, and $\Delta \phi \sim\left(\chi / \chi_{\mathrm{cm}}-1\right)^{1 / 2}$. Then, $\gamma$ and $L$ can be approximately given from eq 6 and 7 as

$$
\begin{gathered}
\gamma \sim k T\left(l / v_{0}\right) N^{1 / 2}\left(\chi / \chi_{\mathrm{cm}}-1\right)^{3 / 2} \\
L \sim l N^{1 / 2}\left(\chi / \chi_{\mathrm{cm}}-1\right)^{-1 / 2}
\end{gathered}
$$

These are the mean-field expressions. In reality, very near the critical point, the mixture becomes more miscible because of large concentration fluctuations, resulting in $\chi_{\mathrm{c}}$ at the real critical point being larger than $\chi_{\mathrm{cm}}$, and the critical exponents $(\mu=1.26$ for $\gamma ; v=0.63$ for $L)$ different from those given by the mean field theory $(\mu=1.5$; $v=0.5){ }^{8,9}$

In Region II, where $2 / N<\chi<1$, the segregation strength is strong, so that one can put $-S_{\max }=2 k T \chi$ and $\Delta \phi=1$, but the entropy term $\kappa_{\mathrm{s}}$ still dominates over the energy term $\kappa_{\mathrm{e}}$, i.e., $\kappa_{\max }=k T l^{2} / 9$. Therefore, eq 6 and 7 yield eq 15 and 16 for this region.

$$
\begin{gathered}
\gamma \sim\left(k T l^{2}\right)^{1 / 2}(k T \chi)^{1 / 2} / v_{0}=\left(k T / v_{0}\right) l \chi^{1 / 2} \sim\left(k T \chi / v_{0}\right) L \\
L \sim\left(k T l^{2}\right)^{1 / 2}(k T \chi)^{-1 / 2}=l \chi^{-1 / 2}
\end{gathered}
$$

The entropy term $k T l^{2}$ can possibly give rise to a positive temperature dependence of $\gamma$ even when the blend has the upper critical solution temperature, namely $\gamma$ may increase with increasing temperature. This prediction was experimentally confirmed in the previous paper. ${ }^{4}$

Region III (very strong segregation, $\chi>1$ ) is not so common in polymeric systems, since most of polymer combinations have a $\chi$-value less than unity. In this region, the energy term dominates over the entropy term, so that, $\kappa_{\max }=k T \chi \lambda^{2} / 2$. Since $-S_{\max }=2 k T \chi$ and $\Delta \phi=1$, eq 6 and 7 yield

$$
\begin{gathered}
\gamma=\left(k T / v_{0}\right) \lambda \chi / 2 \sqrt{2} \sim\left(k T \chi / v_{0}\right) L \\
L=\sqrt{2} \lambda
\end{gathered}
$$

More exact, explicit expressions in the framework of mean-field theory are given for Regions I and II. For the Region I, one has ${ }^{3,7,10}$

$$
\begin{gathered}
\gamma=\frac{2}{3} \frac{k T_{\mathrm{cm}}}{v_{0}} \frac{\bar{l}}{\left(N_{1} N_{2}\right)^{1 / 4}} \varepsilon_{\chi \mathrm{m}}^{3 / 2}=\frac{\sqrt{2}}{3} \frac{k T \chi_{\mathrm{cm}}^{1 / 2} \bar{l}}{v_{0}} \frac{\varepsilon_{\chi \mathrm{m}}^{3 / 2}}{f_{\text {asy }}^{2}} \\
L=\frac{2}{3 \sqrt{2}} \bar{l}\left(N_{1} N_{2}\right)^{1 / 4} \varepsilon_{\chi}^{-1 / 2}=\frac{2}{3} \bar{l}_{\chi}^{-1 / 2} \chi_{\mathrm{cm}}^{-1 / 2} f_{\text {asy }}^{2}
\end{gathered}
$$

with

$$
\begin{gathered}
\varepsilon_{\chi \mathrm{m}}=\frac{\chi}{\chi_{\mathrm{cm}}}-1 \\
\chi_{\mathrm{cm}}=\frac{1}{2}\left(N_{1}^{-1 / 2}+N_{2}^{-1 / 2}\right)^{2} \\
=\frac{2 f_{\mathrm{asy}}^{4}}{\left(N_{1} N_{2}\right)^{1 / 2}} \\
f_{\text {asy }}^{2}=\frac{1}{2}\left[\left(\frac{N_{2}}{N_{1}}\right)^{1 / 4}+\left(\frac{N_{1}}{N_{2}}\right)^{1 / 2}\right] \\
T^{2}=\left[\left(\frac{R_{1}^{2}}{N_{1}}\right) N_{1}^{1 / 2}+\left(\frac{R_{2}^{2}}{N_{2}}\right) N_{2}^{1 / 2}\right] /\left(N_{1}^{1 / 2}+N_{2}^{1 / 2}\right)
\end{gathered}
$$

Here, $\chi_{\mathrm{cm}}$ is the $\chi_{\mathrm{c}}$ of mean-field behavior, and the factor $f_{\text {asy }}$ represents asymmetry of polymeric index.

For the strong segregation region, Region II, the Helfand-Tagami theory gives more quantitative expression at the higher molecular weight limit as ${ }^{11}$

$$
\begin{gathered}
\gamma=\frac{k T \chi^{1 / 2} T}{\sqrt{6} v_{0}} \\
L=\frac{2 \bar{l}}{(6 \chi)^{1 / 2}}
\end{gathered}
$$

which give the numerical front factors in eq 14 and 15 for Region II.

Correction for less strong segregation has been made for these equations by Broseta et al. ${ }^{12}$ to be expressed as

$$
\begin{aligned}
& \gamma=\frac{k T \chi^{1 / 2} T}{\sqrt{6} v_{0}}\left[1-\frac{\pi^{2}}{12 \chi}\left(N_{1}^{-1}+N_{2}^{-1}\right)+\cdots\right] \\
& L=\frac{2 l}{(6 \chi)^{1 / 2}}\left[1-\frac{2 \ln 2}{\chi}\left(N_{1}^{-1}+N_{2}^{-1}\right)\right]^{-1 / 2}
\end{aligned}
$$

Scaled Expression and a Semi-Empirical Expression

Asymptotic expressions (eq 17-20, 25, and 26) in the three regions are summarized in the following scaled forms for mixtures with no strong asymmetry of polymeric indices as

$$
\frac{\gamma}{\gamma^{*}}=\frac{\sqrt{2}}{3}\left(\varepsilon_{\chi \mathrm{m}} / f_{\mathrm{asy}}\right)^{3 / 2} \quad \chi \sim \chi_{\mathrm{cm}} \quad \text { (Region I) }
$$




$$
\begin{aligned}
& =\frac{1}{\sqrt{6}}\left(\varepsilon_{\chi \mathrm{m}} / f_{\text {asy }}\right)^{-1 / 2} \quad 1>\chi>\chi_{\mathrm{cm}}(\text { Region II }) \\
& =\frac{1}{2 \sqrt{2}}(\lambda / \bar{l}) \chi_{\mathrm{cm}}^{1 / 2} f_{\text {asy }}^{1 / 2} \quad \chi>1 \\
& \frac{L}{L^{*}}=\frac{2 \sqrt{2}}{3}\left(\varepsilon_{\chi \mathrm{m}} / f_{\text {asy }}\right)^{-1 / 2} \quad \chi \sim \chi_{\mathrm{cm}} \\
& =\frac{2}{\sqrt{6}}\left(\varepsilon_{\chi \mathrm{m}} / f_{\text {asy }}\right)^{-1 / 2} \quad 1>\chi>\chi_{\mathrm{cm}} \\
& =\sqrt{2}(\lambda / \bar{l}) \chi_{\mathrm{cm}}^{1 / 2} f_{\text {asy }}^{-3 / 2} \quad \chi>1
\end{aligned}
$$

with definitions of characteristic interfacial tension $\gamma^{*}$ and thickness $L^{*}$

$$
\begin{gathered}
\gamma^{*}=\left(\frac{k T}{v_{0}} \chi\right) L^{*} / f_{\text {asy }}^{1 / 2}=\left(\frac{k T}{v_{0}}\right) \bar{l} \chi \chi_{\mathrm{cm}}^{-1 / 2} f_{\text {asy }}^{-1 / 2} \\
L^{*}=\bar{l}_{\chi_{\mathrm{c}}^{-1 / 2}} f_{\text {asy }}^{3 / 2}
\end{gathered}
$$

The scaled $\gamma, \gamma / \gamma^{*}$, and the scaled $L, L / L^{*}$, for Regions I and II are given as system-independent universal functions of the scaled $\varepsilon_{\chi \mathrm{m}}, \varepsilon_{\chi \mathrm{m}} / f_{\text {asy }}$, while those for Region III are not universal ones, but constants depending on the system. The scaled expressions of eq 29 and 30 representing the interfacial tension in Regions I and II as a function of segregation strength are illustrated in Figure 1.

To derive a semi-empirical equation describing the behavior covering over Regions I and II, where we can put $\kappa_{\mathrm{e}}=0$, we start with the approximate expressions for $\gamma$ and $L$, eq 6 and 7 . We have the equations for the quantities $S_{\max }$ and $\kappa_{\max }$ (eq 11 and 12), which can be rewritten as

$$
\begin{gathered}
S_{\mathrm{max}}=2 k T\left(\chi_{\mathrm{cm}}-\chi\right)=-2 k T \chi_{\mathrm{cm}} \varepsilon_{\chi \mathrm{m}} \\
\kappa_{\max }=\frac{k T}{9} l^{2} f_{\mathrm{asy}}^{4}
\end{gathered}
$$

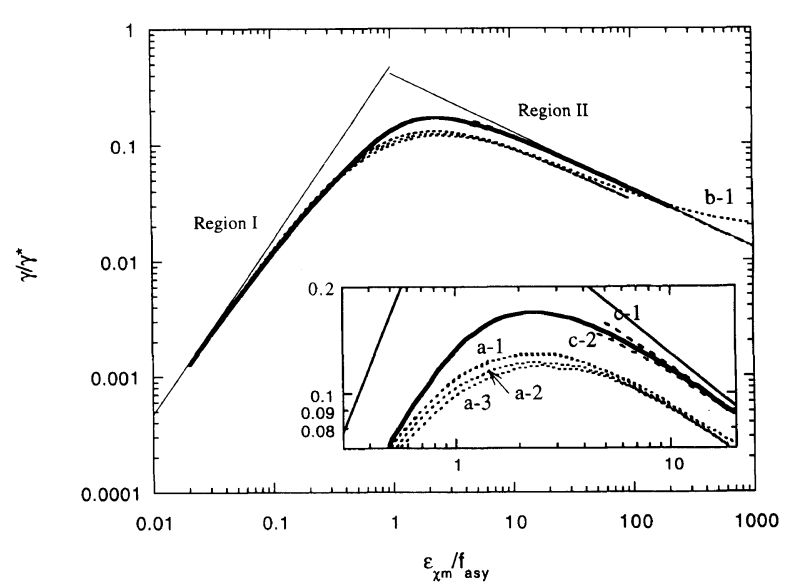

Figure 1. Interfacial tension as a function of segregation strength in the scaled form based on the mean-field theory. Thin solid lines denote asymptotic behavior of respective segregation regions (eq 29 and 30); dotted lines denote the square-gradient theory (eq 3 with eq 8 and 10): $\mathrm{a}, \kappa_{\mathrm{e}}=0$ with $f_{\text {asy }}=1(\mathrm{a}-1), 1.04\left[N_{1} / N_{2}=5\right](\mathrm{a}-2)$, and $1.08\left[N_{1} / N_{2}=\right.$ 10](a-3); b, $\lambda=l / 2, N_{1}=N_{2}=500(\mathrm{~b}-1)$; dashed lines denotes modified Helfand-Tagami theory (eq 50): $f_{\text {asy }}=1(\mathrm{c}-1)$ and 1.08(c-2); and thick solid line denotes semi-empirical equation (eq 48). Inset is an enlarged one for the crossover region.
Now, we need an expression of $\Delta \phi$, and present here the following empirical equation.

$$
(\Delta \phi)^{2}=\left[1-\exp \left\{-\left(3 f_{\text {asy }}^{4} \varepsilon_{\chi \mathrm{m}}\right)^{\alpha}\right\}\right]^{1 / \alpha} \quad(\alpha=0.9)
$$

This equation reproduces the exact mean-field expression near the critical point $\left((\Delta \phi)^{2}=3 f_{\text {asy }}^{4} \varepsilon_{\text {一m }}\right)$, approximates the coexistence curve of symmetrical polymer blends in strong segregation regions $(\Delta \phi=1)$, and gives a good expression for the symmetrical system over the whole range of $\varepsilon_{\chi \mathrm{m}}$ by putting $\alpha=0.9$.

Equation 7 can be transformed by eq 22, 37, and 38 into

$$
L=\frac{2}{3 \sqrt{2}} \bar{l}\left(N_{1} N_{2}\right)^{1 / 4} \varepsilon_{\chi \mathrm{m}}^{-1 / 2}
$$

To make eq 40 be consistent with more exact asymptotic expressions of eq 20 and 26 , the numerical factor $g_{\mathrm{L}}$, which is weakly dependent on $\varepsilon_{\chi \mathrm{m}}$, is defined by

$$
L=g_{\mathrm{L}} \bar{l}\left(N_{1} N_{2}\right)^{1 / 4} \varepsilon_{\chi \mathrm{m}}^{-1 / 2}
$$

and should satisfy

$$
g_{\mathrm{L}}= \begin{cases}2 / 3 & \varepsilon_{\chi \mathrm{m}} \ll 1 \\ (1 / \sqrt{3}) / f_{\text {asy }}^{2} & \varepsilon_{\chi \mathrm{m}} \gg 1\end{cases}
$$

Equations $37-39$ yield the following expression from eq 6 .

$$
\gamma=\frac{L}{4}\left(\frac{k T}{v_{0}}\right) \chi_{\mathrm{c}} \varepsilon_{\chi \mathrm{m}}\left[1-\exp \left\{-\left(3 f_{\text {asy }}^{-4} \varepsilon_{\chi \mathrm{m}}\right)^{\alpha}\right\}\right]^{1 / \alpha}
$$

The numerical factor $g_{\gamma}$ is defined by

$$
\begin{aligned}
\gamma= & g_{\gamma}\left(\frac{k T}{v_{0}}\right) \bar{l} f_{\text {asy }}^{4}\left(N_{1} N_{2}\right)^{-1 / 4} \\
& \times \varepsilon_{\chi \mathrm{m}}^{1 / 2}\left[1-\exp \left\{-\left(3 f_{\text {asy }}^{-4} \varepsilon_{\chi \mathrm{m}}\right)^{\alpha}\right\}\right]^{1 / \alpha}
\end{aligned}
$$

and should have the following values in asymptotic regions so as for eq 44 to be in accordance with the expressions of eq 19 and 25.

$$
g_{\gamma}= \begin{cases}2 / 9 & \varepsilon_{\chi} \ll 1 \\ 1 /\left(\sqrt{3} f_{\text {asy }}^{2}\right. & \varepsilon_{\chi} \gg 1\end{cases}
$$

Simple expressions for $g_{\mathrm{L}}$ and $g_{\gamma}$ respectively satisfying eq 42 and 45 are here assumed to be

$$
g_{\mathrm{L}}=\frac{\frac{2}{3}+\frac{\varepsilon_{\chi \mathrm{m}}^{1 / 2}}{\sqrt{3} f_{\text {asy }}^{2}}}{\left(1+\varepsilon_{\chi \mathrm{m}}\right)^{1 / 2}}
$$

and

$$
g_{\gamma}=\left(\frac{2}{9}+\frac{\varepsilon_{\chi \mathrm{m}}^{2}}{\sqrt{3} f_{\text {asy }}^{2}}\right) /\left(1+\varepsilon_{\chi \mathrm{m}}^{2}\right)
$$

Then, we finally obtain semi-empirical equations for scaled expressions of $\gamma$ and $L$ from eq 44 with eq 47 and eq 41 with eq 46 as

$$
\begin{aligned}
\tilde{\gamma} \equiv & \gamma / \gamma^{*} \\
= & \left(\frac{\sqrt{2}}{9}+\frac{1}{\sqrt{3}}\left(\frac{\varepsilon_{\chi \mathrm{m}}}{f_{\text {asy }}}\right)^{2}\right)\left(\frac{\varepsilon_{\chi \mathrm{m}}}{f_{\text {asy }}}\right)^{1 / 2} \\
& \times \frac{f_{\text {asy }}^{3}}{\left(1+\varepsilon_{\chi \mathrm{m}}^{2}\right)\left(1+\varepsilon_{\chi \mathrm{m}}\right)}\left[1-\exp \left[-\left\{\frac{3}{f_{\text {asy }}^{3}}\left(\frac{\varepsilon_{\chi \mathrm{m}}}{f_{\text {asy }}}\right)\right\}^{\alpha}\right]\right]^{1 / \alpha}
\end{aligned}
$$

Polym. J., Vol. 29, No. 3, 1997 


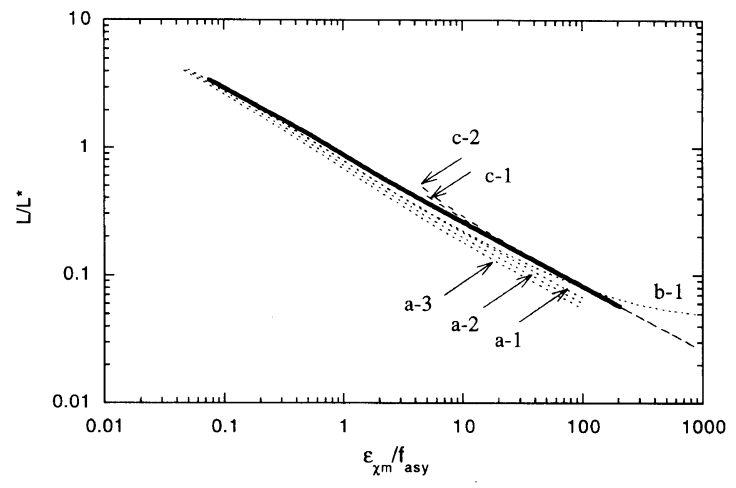

Figure 2. Interfacial thickness as a function of segregation strength in the scaled form based on the mean-field theory. Dotted lines denote the square-gradient theory (eq 4 with eq 8 and 10): a, $\kappa_{\mathrm{e}}=0$ with $f_{\text {asy }}=1(\mathrm{a}-1), \quad 1.04\left[N_{1} / N_{2}=5\right](\mathrm{a}-2)$, and $1.08\left[N_{1} / N_{2}=10\right](\mathrm{a}-3) ; \quad \mathrm{b}$, $\lambda=l / 2, N_{1}=N_{2}=500(\mathrm{~b}-1)$; dashed lines denote modified HelfandTagami theory (eq 51): $f_{\text {asy }}=1(\mathrm{c}-1)$ and $1.08(\mathrm{c}-2)$; and thick solid line denotes semi-empirical equation (eq 49)

$$
\begin{aligned}
& \simeq\left(\frac{\sqrt{2}}{9}+\frac{1}{\sqrt{6}}\left(\frac{\varepsilon_{\chi \mathrm{m}}}{f_{\text {asy }}}\right)^{2}\right)\left(\frac{\varepsilon_{\chi \mathrm{m}}}{f_{\text {asy }}}\right)^{-1 / 2} \\
& \times\left(1+\left(\frac{\varepsilon_{\chi \mathrm{m}}}{f_{\text {asy }}}\right)^{2}\right)^{-1}\left[1-\exp \left\{-3^{\alpha}\left(\frac{\varepsilon_{\chi \mathrm{m}}}{f_{\text {asy }}}\right)^{\alpha}\right\}\right]^{1 / \alpha} \\
& \tilde{L} \equiv L / L^{*} \\
& =\frac{\sqrt{2}}{\sqrt{3}}\left\{\frac{2}{\sqrt{3}}+f_{\text {asy }}^{-3 / 2}\left(\frac{\varepsilon_{\chi}}{f_{\text {asy }}}\right)^{1 / 2}\right\}\left(\frac{\varepsilon_{\chi}}{f_{\text {asy }}}\right)^{-1 / 2}
\end{aligned}
$$

Equations 48 and 49 are shown in Figures 1 and 2, respectively. The equations, eq 27 and 28 , for the strong segregation region with correction of less strong segregation are rewritten in the scaled form as

$$
\begin{aligned}
\frac{\gamma}{\gamma^{*}}= & \frac{1}{\sqrt{6}}\left(\varepsilon_{\chi \mathrm{m}} / f_{\text {asy }}\right)^{-1 / 2} \\
& \times\left[1-\frac{\pi^{2}}{12\left(1+\varepsilon_{\chi \mathrm{m}}\right)}\left(2-\left(2 f_{\text {asy }}^{4}-1\right)^{-2}\right)+\cdots\right] \\
\frac{L}{L^{*}}= & \frac{2}{\sqrt{6}}\left(\varepsilon_{\chi \mathrm{m}} / f_{\text {asy }}\right)^{-1 / 2} \\
& \times\left[1-\frac{2 \ln 2}{\left(1+\varepsilon_{\chi \mathrm{m}}\right)}\left(2-\left(2 f_{\text {asy }}^{4}-1\right)^{-2}\right)\right]^{-1 / 2}
\end{aligned}
$$

and are also drawn in Figures 1 and 2, respectively. Numerical calculations of the square-gradient theory of eq 3 and 4 with eq 8 and 10 were carried out to be shown Figures 1 and 2. The calculated interfacial tension for the case of $\kappa_{\mathrm{e}}=0$, which implies the case of $\chi \ll 1$, accordingly Regions I and II, does not significantly depend on the asymmetry factor in the scaled plots, and approximately satisfies the universality of scaling relation of $\gamma / \gamma^{*} v s . \varepsilon_{\chi \mathrm{m}}$ fairly well as long as the asymmetry is weak, $0.1<N_{1} / N_{2}<10$, i.e., $f_{\text {asy }}<1.08$. Equation 50 also approximately satisfies the universality for $\varepsilon_{\chi \mathrm{m}} /$ $f_{\text {asy }}>50$. The square gradient theory exhibits a little smaller values than the prediction of eq 30 , i.e., eq 25 , in the Region II. The calculated curve for $\lambda=l / 2\left(\kappa_{\mathrm{e}} \neq 0\right)$ with $N_{1}=N_{2}=500$ exhibits the crossover behavior from Region I to Region II around $\varepsilon_{\chi \mathrm{m}}=30$ as expected although the crossover is a very gentle change. The semi-
Table I. Characteristics of polymer samples

\begin{tabular}{clrr}
\hline & Sample code & $M_{w}$ & $M_{w} / M_{n}$ \\
\hline \multirow{2}{*}{ PDMS } & PDMS5 & 460 & $1.0^{\mathrm{a}}$ \\
& PDMS14 & 1400 & 1.10 \\
& PDMS37 & 3700 & 1.24 \\
PS & PS6 & 600 & $<1.10$ \\
& PS22 & 2200 & $<1.10$ \\
\hline
\end{tabular}

a PDMS5 is substantially the pentamer.

empirical equation, eq 48, well agrees with the prediction of the square gradient theory (SGT) in Region I, and well reproduces eq 50 which describes the deviation from the asymptotic behavior in Region II. Equation 48 smoothly connects these two predictions of SGT in Region I and eq 50 in Region II. Changes of interfacial thickness predicted by various calculations exhibit similar relations to each other.

\section{EXPERIMENTAL}

\section{Materials}

Poly(dimethylsiloxane) (PDMS)s were products of Shin-etsu Chemical Co. Ltd. Details of purification and characterization methods have been described elsewhere. ${ }^{13}$ Polystyrene (PS)s were products of Pressure Chemical Co. Sample codes and characteristics of PDMS and PS samples are listed in Table I.

\section{Interfacial Tension Measurements}

Interfacial tension of the phase-separated mixtures was measured in the same way as in the previous study ${ }^{3}$ using sessile-drop method. The apparatus was a specially designed one. The blended sample was sealed in a glass cell and was shaken to be mixed at high temperatures. Then, it was cooled down to a temperature of the measurement. PS-rich phase sank due to its higher density and accumulated on the plate to form a drop in the PDMS-rich upper phase. Image of the drop was focused on the detector of a CCD camera, and its size and shape were measured by image analysis. Temperature was controlled to within $\pm 0.05^{\circ} \mathrm{C}$.

The value of $\gamma / g \Delta \rho$, with $g$ and $\Delta \rho$ being the gravity constant and density difference between coexisting phases, respectively, was evaluated from size and shape of the drop by fitting of the drop profile computed by the Laplace equation. ${ }^{14}$ Interfacial tension $\gamma$ was calculated from the value of $\gamma / g \Delta \rho$ with density difference $\Delta \rho$. The $\Delta \rho$ was evaluated from independently-measured coexistence curves of the PS/PDMS blends. ${ }^{5}$ Details of the experiments and data analysis have been described in the previous paper. ${ }^{3}$

\section{RESULTS AND DISCUSSION}

\section{Temperature Dependence of Interfacial Tension}

Interfacial tensions $\gamma$ for PS/PDMS blends of various molecular weights are plotted against temperature in Figure 3, along with the data of the previous studies. ${ }^{3,4}$ PDMS's with the sample codes of PDMS-1 and PDMS-2 are of higher molecular weights, $M_{w}=2.3 \times 10^{5}$ and $M_{w}=1 \times 10^{5}$, respectively. The data of Koberstein et al. ${ }^{15}$ 


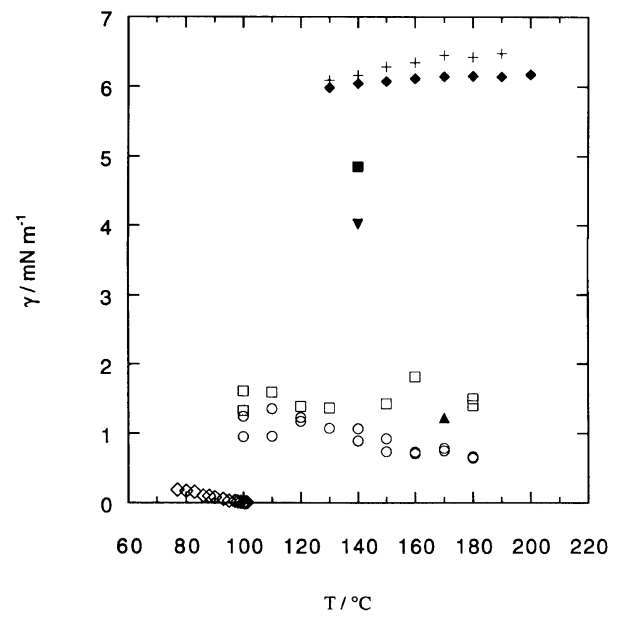

Figure 3. Temperature dependence of interfacial tension $\gamma$ for various molecular-weight PS/PDMS blends. ( $\diamond)$ PS6/PDMS5 ${ }^{3}$; (O) PS6/ PDMS14; ( $\square$ ) PS6/PDMS37; (A) PS22/PDMS5; (+) PSPDMS-1 $\left(M_{w}=2.3 \times 10^{5}\right)^{4} ;(\diamond)$ PDMS-2 $\left(M_{w}=1 \times 10^{5}\right)^{4} ;(\mathbf{\square}, \boldsymbol{\nabla})$ data of Koberstein et al. ${ }^{15}$ for PS $\left(M_{n}=4 \times 10^{3}\right) / \operatorname{PDMS}\left(M_{n}=4.5 \times 10^{3}\right)$ and PS $\left(M_{n}=4 \times 10^{3}\right) /$ PDMS $\left(M_{n}=1.6 \times 10^{3}\right)$, respectively.

for PS4K $\left(M_{n}=4 \times 10^{3}\right) /$ PDMS4.5K $\quad\left(M_{n}=4.5 \times 10^{3}\right)$ and PS4K $\left(M_{n}=4 \times 10^{3}\right) /$ PDMS1.6K $\left(M_{n}=1.6 \times 10^{3}\right)$ are also plotted. As the molecular weight(s) of one or both of components increase(s), the absolute value of $\gamma$ increases, accompanying with decreasing miscibility of the blend. The composition of coexisting phases approaches unity or zero, and the phase separation becomes more complete, as the molecular weight increases. $^{5}$ The oligomer blend (PS6/PDMS5) near the critical point exhibits a rapid increase of $\gamma$ with decreasing temperature, while the high molecular-weight blend show a positive temperature dependence of $\gamma$. The intermediate molecular-weight blends show a negative mild change in PS6/PDMS14 or almost no change in PS6/ PDMS37 with increasing temperature. Namely, as the molecular weight increases, the temperature dependence of $\gamma$ (i.e., the slop of $\gamma-T$ plots) changes from negative to positive in a fixed range of temperature.

\section{Universal Behavior of Interfacial Tension as a Function} of Reduced Temperature Scale

Characteristic interfacial tension $\gamma *$ for scaling (eq 35) of a particular system can be evaluated by the following equation.

$$
\gamma^{*}=R T\left(\frac{\chi}{V_{0}}\right)\left(\frac{V_{0}}{\chi_{\mathrm{cm}}}\right)^{1 / 2} \bar{\rho}^{1 / 2} \overline{\left(\frac{R^{2}}{M}\right)^{1 / 2} / f_{\text {asy }}^{1 / 2}}
$$

The equation was obtained by replacing the average Kuhn-segment length $T$ (see eq 24 ) by an expression in terms of the mean-square end-to-end distance $R^{2}$ divided by molecular weight $M$.

$$
\bar{l}^{2}=\overline{\left(\frac{R^{2}}{M}\right)} \cdot \overline{M_{0}}
$$

with

$$
\overline{\left(\frac{R^{2}}{M}\right)}=\frac{\left(\frac{R_{1}^{2}}{M_{1}}\right) N_{1}^{1 / 2} M_{01}+\left(\frac{R_{2}^{2}}{M_{2}}\right) N_{2}^{1 / 2} M_{02}}{N_{1}^{1 / 2} M_{01}+N_{2}^{1 / 2} M_{02}}
$$

$$
\overline{M_{0}}=\frac{N_{1}^{1 / 2} M_{01}+N_{2}^{1 / 2} M_{02}}{N_{1}^{1 / 2}+N_{2}^{1 / 2}}
$$

Here, $M_{0}$ is the molecular weight of a monomer, $\bar{\rho}=$ $\overline{M_{0}} / V_{0}$ and $V_{0}=v_{0} N_{\mathrm{A}}$ with $N_{\mathrm{A}}$ being the Avogadro constant.

The $\chi$-parameter between PS and PDMS as a function of temperature and molecular weight has been evaluated from the coexistence curve in a previous work, where the $\chi$-parameter was determined such that the Flory-Huggins type mean-field theory reproduced the experimental $\Delta \phi$ of the coexistence curve excluding the vicinity of the critical point. ${ }^{5}$ These are given as $\chi / V_{0} /$ $\left(\mathrm{mol} \mathrm{ml}^{-1}\right)=1.849 / T(\mathrm{~K})-0.00143$ for PS6/PDMS5, $\chi / V_{0} /\left(\mathrm{mol} \mathrm{m}^{-1}\right)=1.527 / T(\mathrm{~K})-0.00063$ for PS6/ PDMS14, $\chi / V_{0} /\left(\mathrm{mol} \mathrm{ml}^{-1}\right)=2.027 / T(\mathrm{~K})-0.00164$ for PS6/PDMS37, $\chi / V_{0} /\left(\mathrm{mol} \mathrm{ml}^{-1}\right)=2.446 / T(\mathrm{~K})-0.00213$ for PS6/PDMS2K, $\chi / V_{0} /\left(\mathrm{mol} \mathrm{ml}^{-1}\right)=2.372 / T(\mathrm{~K})-$ 0.00193 for PS22/PDMS5, and $\chi / V_{0} /\left(\mathrm{mol} \mathrm{ml}^{-1}\right)=$ $2.940 / T(\mathrm{~K})-0.00247$ for PS/PDMS of high $M_{w}$. For the systems of the study by Koberstein et al., $\left(\chi / V_{0}\right) / \mathrm{g} \mathrm{ml}^{-1}$ was estimated by extrapolating the above equations to be 0.00448 and 0.00437 at $140^{\circ} \mathrm{C}$ for PS4K/PDMS4.5K and PS4K/PDMS1.6K, respectively. The mean density $\bar{\rho}$ were evaluated from the specific volume of pure PS and $\mathrm{PDMS}^{5}$ under the approximation of the additivity of the volume. The value of $\left(R^{2} / M\right)^{1 / 2}$ is accidentally the same for both of PS and PDMS. ${ }^{16}$ Values of $\left(R^{2} / M\right)^{1 / 2}$ were calculated from data in literatures ${ }^{16}$ to be $\left(R^{2}\right.$ $M)^{1 / 2}=0.067 \times(1+0.0003 \times(t-140)) \mathrm{nm} \mathrm{mol}^{-1 / 2} \mathrm{~g}^{1 / 2}$ as a function of temperature $t$ in ${ }^{\circ} \mathrm{C}$. The polymerization index $N$ is defined to be proportional to the molar volume, and can be evaluated from the molar mass and specific volume. $\chi_{\mathrm{cm}} / V_{0} /\left(\mathrm{mol} \mathrm{ml}^{-1}\right)$ is given as the critical value with $N$ being taken to be the molar mass itself in eq 22. The asymmetric factor $f_{\text {asy }}$ was evaluated from the ratio of $N_{1} / N_{2}$ by the definition of eq 23. The reduced distance of $\chi$ from the mean-field critical point, $\varepsilon_{\chi \mathrm{m}}$, was calculated from $\chi / V_{0}$ and $\chi_{\mathrm{cm}} / V_{0}$ evaluated above.

All experimental data presented in Figure 3 are plotted in the scaled form of $\gamma / \gamma^{*} v s . \varepsilon_{\chi \mathrm{m}} / f_{\text {asy }}$ in Figure 4. The present experimental data are located in the intermediate region between Regions I and II, and closer to Region I, while the data of Koberstein et al. closer to Region II.

These plots are reasonably bridging the two asymptotic regions of I and II in the scaled relation. The semiempirical equation (eq 48) presented here describes the experimental data fairly well, although the calculated curve predicts a little larger values than the experimental data around $\varepsilon_{\chi \mathrm{m}}=1$.

The oligomer blend (PS6/PDMS5) is weakly segregated, showing the critical point. Because of large concentration fluctuations near the critical point, $\gamma$ follows the power law with the exponent $\mu=1.27,{ }^{3}$ and deviated away from the mean-field prediction as $\varepsilon_{\gamma \mathrm{m}}$ decreases. With increasing $\varepsilon_{x \mathrm{~m}}, \gamma$ approaches the mean-field behavior.

In evaluating the semi-empirical equation by comparing with the present experimental results, we have to note that the present blend systems may not sufficiently satisfy the basic assumptions imposed in the theoretical consideration. PS/PDMS blends have rather large values of $\chi$-parameter, ${ }^{5}$ so that the condition, $\chi \ll 1$, for Regions 


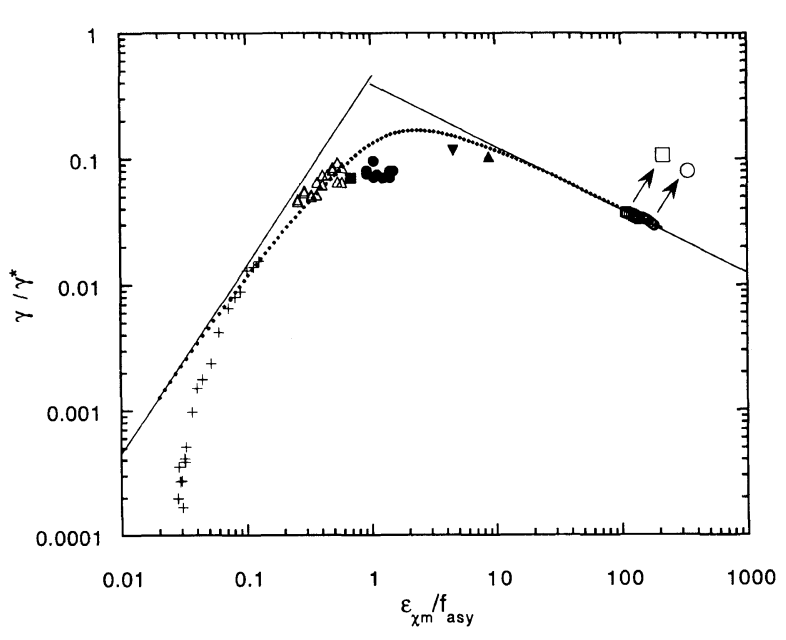

Figure 4. Scaled plots of interfacial tension $\gamma$ against segregation

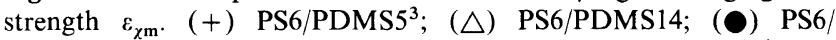
PDMS37; ( PS22/PDMS5; (O) PSPDMS-1 $\left(M_{w}=2.3 \times 10^{5}\right)^{4} ;(\square)$ PDMS-2 $\left(M_{w}=1 \times 10^{5}\right)^{4},(\mathbf{\Delta}, \boldsymbol{\nabla})$ data of Koberstein et al ${ }^{15}$ for PS $\left(M_{n}=4 \times 10^{3}\right) /$ PDMS $\left(M_{n}=4.5 \times 10^{3}\right)$ and PS $\left(M_{n}=4 \times 10^{3}\right) /$ PDMS $\left(M_{n}=1.6 \times 10^{3}\right)$, respectively. Thin solid lines denote asymptotic behavior in respective segregation regions (eq 29 and 30); and thick dotted line denotes semi-empirical equation (eq 48).

I and II is not safely satisfied to neglect the term of $\kappa_{\mathrm{e}}$ in $\kappa$ of eq 10. More explicit expression of the condition for the neglect is that $\chi \ll(2 / 9)(l / \lambda)^{2}$. In the present blends, the value of $\chi$ for the PS monomer size ranges from 0.28 to $0.46,{ }^{5}$ and may not be sufficiently smaller than $(2 / 9)(l / \lambda)^{2}$, which is about unity in the case of $l=2 \lambda$. Another question for applicability of the theory is that the theory assumes a blend of Gaussian-chain polymers, while the present blends involve short-chain oligomers, as already pointed out in the previous study. ${ }^{3}$ In view of these points, the term $\kappa$ in the present comparison of experimental and calculated results is somewhat phenomenological. Therefore, in order to criticize the theoretical treatment more quantitatively, we have to choose more suitable blend systems, which should be blends of higher molecular weight with weak interactions (i.e., smaller values of $\chi$ ).

\section{REFERENCES}

1. T. Nose, J. Jpn. Soc. Colour Mater. (in Japanese), 64, 729 (1991).

2. G. H. Fredrickson, in "Physics of Polymer Surfaces and Interfaces," I. C. Sanchez, Ed., Butterworth-Heinemann, Oxford, 1992, Chapter 1.

3. T. Nose, Macromolecules, 28, 3702 (1995).

4. T. Nose and T. Kasemura, J. Adhesion, 59, 207 (1996).

5. T. Nose, Polymer, 36, 2243 (1995).

6. J. W. Cahn and J. E. Hilliard, J. Chem. Phys., 28, 258 (1958).

7. T. Nose, Polym. J., 8, 96 (1976).

8. B. Widom, J. Chem. Phys., 43, 3892 (1965).

9. H. E. Stanley, in "Introduction to Phase Transitions and Critical Phenomena," Clarendon Press, Oxford, 1941, Chapters I and II.

10. J. F. Joanny and L. Leibler, J. Phys. (Paris), 39, 951 (1978)

11. E. Helfand and Y. Tagami, J. Chem. Phys., 56, 3592 (1971).

12. E. Broseta, G. H. Fredrickson, E. Helfand, and L. Leibler Macromolecules, 23, 132 (1990)

13. T. Kubo and T. Nose, Polym. J., 24, 1351 (1992).

14. A. W. Adamson, in "Physical Chemistry of Surfaces," 5th ed, John Wiley \& Sons, Inc., New York, N.Y., 1990, Chapter II.

15. W. Hu, J. T. Koberstein, J. P. Lingelser, and Y. Gallot, Macromolecules, 28, 5209, (1995).

16. J. Brandrup and E. H. Immergut, Ed., "Polymer Handbook," 3rd ed, John Weily \& Sons, Inc., New York, N.Y., 1989, VII/33. 